\title{
FLEXURAL VIBRATION OF COUPLED DOUBLE-WALLED CARBON NANOTUBES CONVEYING FLUID UNDER THERMO-MAGNETIC FIELDS BASED ON STRAIN GRADIENT THEORY
}

\author{
Ali Ghorbanpour Arani, Pedram Dashti, Saeed Amir, Mohsen Yousefi \\ Faculty of Mechanical Engineering, Institute of Nanoscience and Nanotechnology, University of Kashan, Kashan, Iran \\ e-mail:aghorban@kashanu.ac.ir; pedi_dashti@yahoo.com; samir@kashanu.ac.ir;mohsen_r.yoosefi@yahoo.com
}

\begin{abstract}
The flexural vibration stability of a coupled double-walled viscoelastic carbon nanotube conveying a fluid based on the Timoshenko beam (TB) model is investigated. The coupled system is surrounded by an elastic medium which is simulated as Pasternak foundation. Van der Waals (vdW) forces between the inner and outer CNTs are taken into account based on the Lenard-Jones model. Using small scale theories, Hamilton's principle and applying two dimensional (2D) magnetic field higher order governing equations are derived. The differential quadrature method (DQM) is applied to solve partial differential equations and investigate natural frequency of the system. The effects of viscoelastic constant, magnetic field with variable magnitudes and surface stresses on natural frequency of the structure are demonstrated in this study.
\end{abstract}

Keywords: flexural vibration, conveying fluid, coupled-system, thermo-magnetic fields, viscoelasticity

\section{Introduction}

Since Iijima (1991) discovered Carbon nanotubes (CNTs) many articles have been published in different fields of physics and engineering, which were focused on the CNTs and their applications. For example Ke and Wang (2011) demonstrated the influence of the length scale parameter of modified couple stress theory (MCST) on natural frequency of the double-walled Carbon nanotube (DWCNT). Pradhan and Mandal (2013) investigated the effects of environmental temperature changes on buckling, bending and vibrational stability of the CNT embedded by pinned boundary conditions. Kong et al. (2009) illustrated the effects of strain gradient theory (SGT) on the static, dynamic and free vibration response of a microstructure including CNTs. Kiani (2014) presented a single-walled Carbon nanotube (SWCNT) structure introduced to a 3D magnetic field. He investigated the effects of the magnetic field on longitudinal, transverse and lateral frequencies of the structure. According to his results, longitudinal magnetic field was more effective than the transverse one. Lei et al. (2013) investigated vibration characteristics of nonlocal viscoelastic nanobeams using the Kelvin-Voigt viscoelastic model based on TB theory. They discussed the effects of the Kelvin-Voigt coefficient, nonlocal constant, external damping ratio, and beam length-to-diameter ratio on natural frequencies of the carbon nanotubes. Ansari et al. (2014) illustrated nonlinear free vibration of a TB with different boundary conditions. They used the Gurtin-Murdoch continuum elasticity model to obtain equilibrium equations which were affected by surface stress layers. According to their results, the effects of rigidity of the surface layers and surface residual stress on natural frequency were not neglegible. Lei et al. (2012) illustrated the effects of surface elasticity modulus, residual surface stress, nonlocal parameter and aspect ratio on the transverse natural frequency. Xu et al. (2010) presented the equilibrium equations of a pipe conveying fluid with the pined-pined boundary condition in 
order to investigate the effects of the fluid flow velocity on the natural frequency. The results were obtained using Galerkin's method and a complex mode approach. Ghorbanpour Arani et al. (2014) presented a mathematical model of a coupled viscoelastic CNT conveying fluid flow based on Euler-Bernoulli beam theory affected by Visco-Pasternak foundation, nonlocal small scale theory, surface stresses and a longitudinal magnetic field. They depicted the effects of surface stresses, viscoelastic constant, nonlocal small scale coefficient and the magnetic field on natural frequency of the system. Khosrozadeh and Hajabasi (2012) investigated the natural frequency of a DWCNT including nonlinear vdW interaction and simply supported, fixed or free boundary conditions. The results revealed that the influence of nonlinear components of vdW forces on natural longitudinal frequency was neglected.

Shen and Zhang (2011) presented post-buckling, nonlinear bending and nonlinear vibration analysis of a nanoscale structure based on nonlocal small scale theory. The system including a thermo-elastic CNT rested on an elastic foundation. The effects of the nonlocal parameter and temperature changes on natural frequency, static bending and buckling load were demonstrated in their work. Ghorbanpour Arani and Amir (2013) investigated free vibration analysis of a coupled-Boron nitride nanotube (BNNT) structure affected by electro-thermal fields. The displacement field and strain-stress relation were based on Euler-Bernoulli beam theory and strain gradient theory, respectively. The effects of fluid velocity, aspect ratio and temperature changes on natural frequencies were demonstrated in their results.

In this study, a double-bonded DWCNT conveying viscous fluid flow is presented. The viscoelastic structure is affected by a surface stress layer, 2D magnetic field, vdW interaction and Pasternak foundation loads. The CNTs are based on Timoshenko beam theory, and the governing equations will be obtained using Hamilton's principle. DQM is used to solve the partial differential equations and investigate natural frequency of the system. Finally, the effects of viscoelastic constant, small scale coefficients, surface stresses and the magnetic field on the natural frequency of the structure are presented in this paper.

\section{Governing equations}

\subsection{TB theory}

Figure 1 demonstrates a coupled-structure made of carbon nanotubes based on TB theory in which $L$ is length of the tubes and $R$ and $h$ are outer radius and thickness of the CNTs, respectively. The effects of zigzag graphene sheet rolling procedure are considered in this study. In the zigzag rolling procedure, the radius of CNT is obtained by $R=0.142 p \sqrt{3} / 2 \pi \mathrm{nm}$ in which $P$ denotes the numbers of carbon atoms (Shen and Zhang, 2011). Since the displacement field is based on Timoshenko beam theory, Eq. (2.1) demonstrates strain-displacement relation of TB theory

$$
\varepsilon_{x x i j}=\frac{\partial U_{i j}(x, t)}{\partial x}+z \frac{\partial \Psi_{i j}(x, t)}{\partial x} \quad \gamma_{x z i j}=2 \varepsilon_{x z i j}=\frac{\partial W_{i j}(x, t)}{\partial x}+\Psi_{i j}(x, t)
$$

where the subscript $i=1,2$ indicate the number of upper and lower nanotubes, respectively, $j=1,2$ indicate the inner and outer tubes, respectively, and $\Psi_{i j}(x, t)$ is rotation of cross-section of the nanotubes.

\subsection{Surface stress effect}

Influence of the surface effect on stability of nano or microstructures cannot be ignored because in these structures the surface-to-bulk ratio will increase. An appropriate theoretical notion is offered by Gurtin-Murdoch. It is based on the continuum mechanical model including 


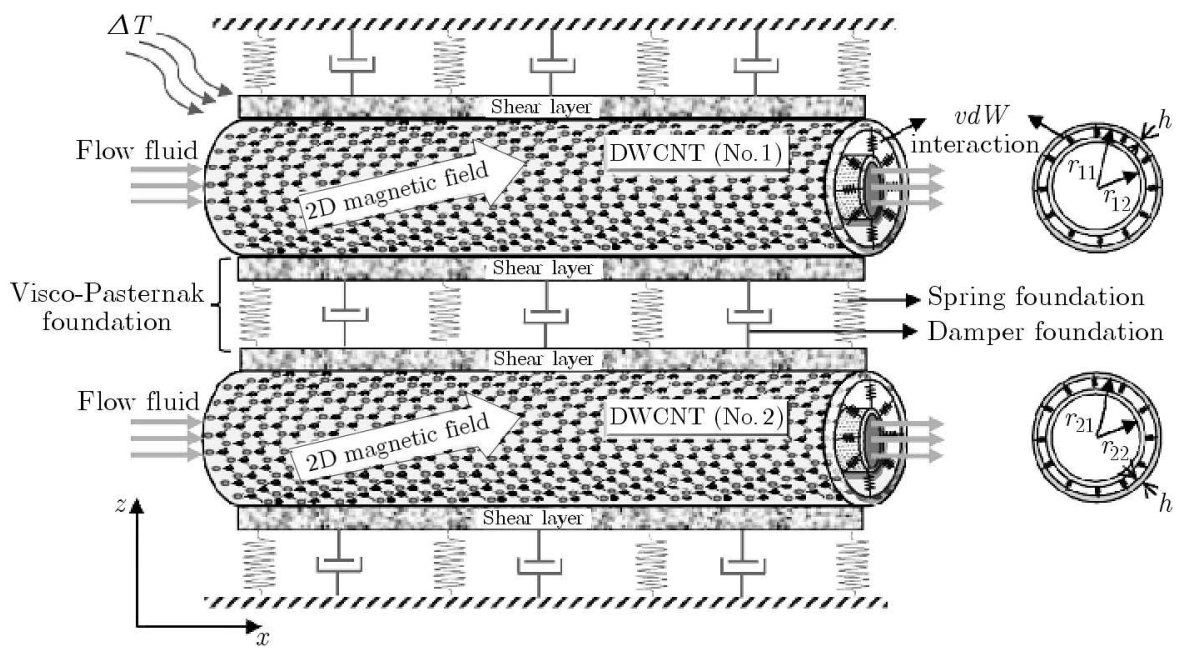

Fig. 1. Schematic of a coupled CNT's deformed element based on the TB model conveying fluid under two dimensional magnetic field

surface stress effects which is well known as the Gurtin-Murdoch model (Ansari et al., 2014; Lu et al., 2006). Mechanical stresses regarding the TB theory are

$$
T_{x x i j}^{s}=\left(\lambda^{s}+2 \mu^{s}\right) \varepsilon_{x x i j}+\tau^{s} \quad T_{x z i j}^{s}=\tau^{s} \frac{\partial W_{i j}}{\partial x}
$$

where $\tau^{s}$ and $\delta_{n m}$ are the residual surface stress and Kronecker tensor, respectively, and $\lambda^{s}$ and $\mu^{s}$ are surface Lame constants. In this study, we consider that the surface stresses on the layers satisfy the equilibrium relation so $T_{z z i j}^{s}$ cannot be neglected (Lei et al., 2012; Lu et al., 2006), thus the components of stress tensor for the bulk of nanotube can be shown in Eq. (2.3) ${ }_{1}$ (Ansari et al., 2014; Lu et al., 2006)

$$
\begin{aligned}
& T_{x x i j}=E^{*} \varepsilon_{x x}+\frac{\nu}{1-\nu} \sigma_{z z}=E^{*}\left(\frac{\partial U_{i j}}{\partial x}+z \frac{\partial \Psi_{i j}}{\partial x}\right)+\frac{2 z \nu}{h(1-\nu)}\left(\tau^{S} \frac{\partial^{2} W_{i j}}{\partial x^{2}}-\rho^{S} \frac{\partial^{2} W_{i j}}{\partial t^{2}}\right) \\
& T_{x z i j}=G \kappa_{S} \gamma_{x z i j}=G^{*} k_{s}\left(\frac{\partial W_{i j}}{\partial x}+\Psi_{i j}\right)
\end{aligned}
$$

where $\rho^{s}$ is density of the surface layers. $E^{*}=E(1+g \partial / \partial t)$ and $G^{*}=G(1+g \partial / \partial t)$ are viscoelastic parameters of the CNTs based on the Kelvin-Voigt model (Lei et al., 2013). E and $G$ are elasticity and shear modulus of the CNTs, respectively, which are functions of temperature according to Table 1.

\subsection{Hamilton's principle}

To develop a comprehensive model for coupled DWCNT, we use extended Hamilton's principle which can be expressed as follows (Ghorbanpour Arani and Amir, 2013)

$$
\int_{t_{0}}^{t_{1}} \delta \Pi d t=\int_{t_{0}}^{t_{1}} \delta\left(U^{\text {strain }}-K^{\text {Total }}-\Omega^{\text {Total }}\right) d t=0
$$

where $\Pi$ indicates total mechanical energy of the structure which includes kinetic energy $K^{\text {Total }}$, external work done by external forces $\Omega^{\text {Total }}$ and potential energy $U^{\text {strain }}$. 


\subsubsection{Total kinetic energy}

The total kinetic energy of the coupled system contains kinetic energies of nanotubes, surface layer and flow fluid as follows (Ansari et al., 2014; Ghorbanpour Arani et al., 2014)

$$
\begin{aligned}
& K^{\text {Total }}=K_{\text {nanotubes }}+K_{\text {nanotubes }}^{\text {surface }}+K_{\text {fluid }} \\
& =\frac{1}{2} \rho_{f} \int_{0}^{l}\left\{\int_{A_{f}}\left[\left(\frac{\partial U_{i 1}}{\partial t}+z \frac{\partial \Psi_{i 1}}{\partial t}+U_{f} \cos \theta\right)^{2}+\left(\frac{\partial W_{i 1}}{\partial t}-U_{f} \sin \theta\right)^{2}\right] d A\right\} d x \\
& +\frac{\rho^{S}}{2} \int_{0}^{l}\left\{\oint_{S_{i j}}\left[\left(\frac{\partial U_{i j}}{\partial t}+z \frac{\partial \Psi_{i j}}{\partial t}\right)^{2}+\left(\frac{\partial W_{i j}}{\partial t}\right)^{2}\right] d S\right\} d x \\
& +\frac{1}{2} \int_{0}^{l}\left\{\int_{A_{i j}} \rho_{C N T}\left[\left(\frac{\partial U_{i j}}{\partial t}+z \frac{\partial \Psi_{i j}}{\partial t}\right)^{2}+\left(\frac{\partial W_{i j}}{\partial t}\right)^{2}\right] d A_{i j}\right\} d x
\end{aligned}
$$

where $U_{f}$ is the fluid flow velocity and $\rho_{f}, \rho^{S}, \rho_{C N T}$ are density of the fluid, surface layer and CNT, respectively.

\subsubsection{External work}

The external work that includes the elastic medium, thermal and magnetic fields, vdW interactions, and conveying fluid forces are presented as follows (Ghorbanpour Arani et al., 2014)

$$
\begin{aligned}
& \Omega^{\text {Total }}=\underbrace{\left.\frac{1}{2} \int_{0}^{l}\left[\vec{M}_{y}^{L} \Psi_{i j}\right)+\left(\vec{F}_{x}^{L} U_{i j}\right)+\left(\vec{F}_{z}^{L} W_{i j}\right)\right] d x}_{\text {Lorentz work }}+\frac{1}{2} \underbrace{\int_{0}^{l}\left(q_{i j}^{v d W} W_{i j}+q_{i 2}^{\text {Pasternak }} W_{i 2}\right) d x}_{\text {External work done by vdW \& Pasternak }} \\
& +\underbrace{\frac{1}{2} \int_{0}^{l}-E^{*} A_{i j} \alpha \Delta T\left(\frac{\partial W_{i j}}{\partial x}\right)^{2} d x}_{\text {External work done by thermal changes }}+\underbrace{\frac{1}{2} \int_{0}^{l}\left[-\rho_{f} U_{f}^{2} A_{f} \frac{\partial^{2} W_{i 1}}{\partial x^{2}}\left(\cos \theta W_{i 1}+\sin \theta U_{i 1}\right)\right] d x}_{\text {External work done by centripetal fluid force }} \\
& -\underbrace{\frac{1}{2} \int_{0}^{l}\left[\mu_{e} A_{f} \frac{\partial^{3} U_{i 1}}{\partial x^{2} \partial t}+\mu_{e} A_{f} U_{f}\left(-\sin \theta \frac{\partial^{2} \theta}{\partial x^{2}}-\left(\frac{\partial \theta}{\partial x}\right)^{2} \cos \theta\right)\right] U_{i 1} d x}_{\text {External work done by viscous fluid }} \\
& -\underbrace{\frac{1}{2} \int_{0}^{l}\left[\mu_{e} A_{f} \frac{\partial^{3} W_{i 1}}{\partial x^{2} \partial t}+\mu_{e} A_{f} U_{f}\left(-\cos \theta \frac{\partial^{2} \theta}{\partial x^{2}}-\left(\frac{\partial \theta}{\partial x}\right)^{2} \sin \theta\right)\right] W_{i 1} d x}
\end{aligned}
$$

in which the Lorentz external work is the resultant of two-dimensional (2D) magnetic fields applied along the longitudinal and transverse directions. $\vec{H}=H_{x} \vec{i}+H_{z} \vec{k}$ is considered in order to show the effects of this type of magnetic field on stability of our structure. In order to show how the electric and magnetic fields are generated and changed by each other, Maxwell's equations are presented (Ghorbanpour Arani et al., 2014; Kiani, 2014). The load inserted by Pasternak foundation which surrounds the external CNTs and the vdW interaction forces are distributed transverse loads, whose their external works are presented in Eq. (2.7), see Ghorbanpour Arani and Amir (2013), Khosrozadeh and Hajabasi (2012), Shen and Zhang (2011). The structure 
is introduced to a uniform thermal field. Hence, temperature changes apply an axial compressive load to the system. The external work done by the thermal load is shown in the above equation. Finally, the external work done by the viscous fluid flow is presented in Eq. (2.6), see Ghorbanpour Arani et al. (2014), Ghorbanpour Arani and Amir (2013), in which $\mu_{e}$ is the effective viscosity which is modified by the Knudsen number (Ghorbanpour Arani et al., 2014). The Knudsen number is a dimensionless ratio which is used to modify the fluid flow velocity as follows

$$
V C F=\frac{V_{\text {ave }, \text { slip }}}{V_{\text {ave }, \text { no-slip }}}=(1+b \mathrm{Kn})\left(1+4 \frac{2-\sigma_{\nu}}{\sigma_{\nu}} \frac{\mathrm{Kn}}{1+\mathrm{Kn}}\right)
$$

where $V C F$ is the correction factor which will be used to modify the fluid flow velocity as $V_{a v g, s l i p}=V C F \cdot V_{a v g, n o-s l i p}$ and the governing equations as $U_{f}=V_{a v g, s l i p}$.

\subsubsection{Strain energy based on Strain gradient theory}

The strain energy of mechanical structures is related to the stress and strain tensors of each structure. Strain gradient and modified couple stress theories are presented in this article in order to obtain strain energy of coupled DWCNT.

In the constitutive equations of the strain gradient theory (Ghorbanpour Arani and Amir, 2013; Kong et al., 2009), for isotropic linear elastic materials there are only three independent higher-order material length scale parameters in addition to the two classical material parameters. Strain stress potential energy of the bulk and surface layer of the system is obtained by strain gradient theory, and will be investigated as below in the general form

$$
U=\frac{1}{2}\left(\int_{\Gamma}\left(T_{k p} \varepsilon_{p q}+p_{k} \gamma_{k}+\tau_{p t q}^{(1)} \eta_{p t q}^{(1)}+m_{k p} \chi_{k p}\right) d V+\oint_{S}\left(T_{x x}^{S} \varepsilon_{x x}+T_{x z}^{S} \gamma_{x z}\right) d S\right)
$$

in which

$$
\begin{aligned}
\varepsilon_{p q} & =\frac{1}{2}\left(\bar{D}_{p, q}+\bar{D}_{q, p}\right) \quad \gamma_{k}=\varepsilon_{m m, k} \quad \chi_{k p}=\frac{1}{2}\left(\phi_{k, p}+\phi_{p, k}\right) \\
\eta_{p t q}^{(1)} & =\frac{1}{2}\left(\varepsilon_{t q, p}+\varepsilon_{q p, t}+\varepsilon_{p t, q}\right)-\frac{1}{15}\left[\delta_{p t}\left(\varepsilon_{m m, q}+2 \varepsilon_{m q, m}\right)\right] \\
& -\frac{1}{15}\left[\delta_{t q}\left(\varepsilon_{m m, p}+2 \varepsilon_{m p, m}\right)+\delta_{q p}\left(\varepsilon_{m m, t}+2 \varepsilon_{m t, m}\right)\right]
\end{aligned}
$$

where $\varepsilon, \gamma, \boldsymbol{\eta}$ and $\chi$ are strain, dilatation gradient, deviatoric stretch gradient and symmetric rotation gradient tensors, respectively. Stresses and higher order stress tensors are mentioned by

$$
T_{p g}=\left\{\begin{array}{lll}
E^{*} \varepsilon_{p q} & \text { if } & p \equiv q \\
2 \kappa_{S} G^{*} \varepsilon_{p q} & \text { if } & p \neq q
\end{array}\right.
$$

and

$$
p_{k}=2 G^{*} l_{0}^{2} \gamma_{k} \quad \tau_{p t q}^{(1)}=2 G^{*} l_{1}^{2} \eta_{p t q}^{(1)} \quad m_{k p}=2 G^{*} l_{2}^{2} \chi_{k p}
$$

where $\mathbf{T}, \mathbf{p}, \boldsymbol{\tau}$ and $\mathbf{m}$ are stress, higher order stress and deviatoric stress tensors, respectively. According to Eqs. (2.11), $l_{0}, l_{1}$ and $l_{2}$ are parameters of small scale theory. Therefore, with respect to Eqs. (2.5), (2.6) and using Hamilton's principle based on strain gradient theory, equations of motion are obtained. Using dimensionless parameters (Ghorbanpour Arani and Amir, 2013; Kong et al., 2009), we have 


$$
\begin{array}{lccc}
\left(w_{i j}, u_{i j}\right)=\frac{\left(W_{i j}, U_{i j}\right)}{R_{o}} & \zeta=\frac{x}{l} & \bar{L}=\frac{L}{l} & \left(\bar{L}_{0}, \bar{L}_{1}, \bar{L}_{2}\right)=\frac{\left(L_{0}, L_{1}, L_{2}\right)}{L} \\
g^{*}=\frac{g}{l} \sqrt{\frac{E}{\rho_{C N T}}} \quad \tau=\frac{t}{l} \sqrt{\frac{E}{\rho_{C N T}}} & \bar{I}_{i j}=\frac{I_{i j}}{A l^{2}} & u_{f}=\sqrt{\frac{\rho_{f}}{E}} U_{f} \\
H_{x}^{*}=\sqrt{\frac{\eta_{m} H_{x}^{2}}{E}} \quad H_{z}^{*}=\sqrt{\frac{\eta_{m} H_{z}^{2}}{E}} \quad \bar{\mu}=\frac{\mu}{R_{0} \sqrt{E \rho_{f}}} & \beta_{i j}=\frac{\kappa_{s} G A_{i j}}{E A_{i j}} \\
\bar{h}_{1}=\frac{\pi\left(R_{i}^{3}+R_{o}^{3}\right) E_{s} T_{s}}{E A l^{2}} & \bar{h}_{2}=\frac{2 \pi\left(R_{i}+R_{o}\right) E_{s} T_{s}}{E A} & \bar{h}_{3}=\frac{2 \nu \pi I \tau^{s} R_{o}}{(1-\nu) E A h l^{3}} \\
\bar{h}_{4}=\frac{2 \nu \pi I E \rho^{s}}{(1-\nu) E A h \rho_{C N T} l^{2}} & \bar{h}_{5}=\frac{2 \pi \rho^{s}\left(R_{\text {in }}+R_{\text {out }}\right)}{\rho_{C N T} A_{11}} & \bar{h}_{6}=\frac{\pi\left(R_{\text {in }}^{3}+R_{\text {out }}^{3}\right) \rho^{s}}{\rho_{C N T} A_{\text {in }} l^{2}} \\
\left(\bar{c}_{i j}, \bar{c}_{i j}^{\prime}\right)=\frac{\left(c, c^{\prime}\right) l^{2}}{E A_{i j}} & \bar{G}_{p}=\frac{G_{p}}{E A} \quad \bar{K}_{w}=\frac{K_{w} l^{2}}{E A} & \lambda=\frac{l}{R_{o}} \\
\bar{\Pi}_{0}=\frac{2 \pi\left(R_{i}+R_{o}\right) \tau^{s}}{E A} & f=\frac{A_{f}}{A} & \bar{\rho}=\frac{\rho_{f}}{\rho_{C N T}} & \overline{\Delta T}=\alpha \Delta T
\end{array}
$$

Therefore, the dimensionless equilibrium equations can be written as:

— for $\delta u_{i j}=0$

$$
\begin{aligned}
- & \frac{1}{\lambda} \frac{\partial^{2} u_{i j}}{\partial \zeta^{2}}-\frac{g^{*}}{\lambda} \frac{\partial^{3} u_{i j}}{\partial \zeta^{2} \partial \tau}+\frac{2 \bar{L}_{0} \beta_{i j}}{\kappa_{s} \lambda} \frac{\partial^{4} u_{i j}}{\partial \zeta^{4}}+\frac{2 \bar{L}_{0} g^{*} \beta_{i j}}{\kappa_{s} \lambda} \frac{\partial^{5} u_{i j}}{\partial \zeta^{4} \partial \tau}+\frac{20 \bar{L}_{1} \beta_{i j}}{25 \kappa_{s} \lambda} \frac{\partial^{4} u_{i j}}{\partial \zeta^{4}} \\
& +\frac{20 \bar{L}_{1} g^{*} \beta_{i j}}{25 \kappa_{s} \lambda} \frac{\partial^{5} u_{i j}}{\partial \zeta^{4} \partial \tau}+\frac{H_{x}^{*} H_{z}^{*}}{\lambda} \frac{\partial^{2} w_{i j}}{\partial \zeta^{2}}-\frac{H_{z}^{* 2}}{\lambda} \frac{\partial^{2} u_{i j}}{\partial \zeta^{2}}-\frac{\bar{h}_{2}}{\lambda} \frac{\partial^{2} u_{i j}}{\partial \zeta^{2}}+\frac{1}{\lambda} \frac{\partial^{2} u_{i j}}{\partial \tau^{2}} \\
& +\frac{\Gamma_{i j} f_{i j} \bar{\rho}}{\lambda} \frac{\partial^{2} u_{i j}}{\partial \tau^{2}}-\frac{\bar{\mu} \sqrt{\bar{\rho}}}{\lambda} \frac{\partial^{3} u_{i j}}{\partial \tau \partial \zeta^{2}}=0
\end{aligned}
$$

- for $\delta w_{i j}=0$

$$
\begin{aligned}
- & \beta_{i j} \frac{\partial \Psi_{i j}}{\partial \zeta}-\beta_{i j} g^{*} \frac{\partial^{2} \Psi_{i j}}{\partial \zeta \partial \tau}-\frac{\beta_{i j} g^{*}}{\lambda} \frac{\partial^{3} w_{i j}}{\partial \zeta^{2} \partial \tau}-\frac{\beta_{i j}}{\lambda} \frac{\partial^{2} w_{i j}}{\partial \zeta^{2}}+\frac{8 \beta_{i j} \bar{L}_{1}^{2}}{15 \lambda \kappa_{s}} \frac{\partial^{4} w_{i j}}{\partial \zeta^{4}}+\frac{16 \beta_{i j} \bar{L}_{1}^{2}}{15 \kappa_{s}} \frac{\partial^{3} \Psi_{i j}}{\partial \zeta^{3}} \\
+ & \frac{8 \beta_{i j} g^{*} \bar{L}_{1}^{2}}{15 \lambda \kappa_{s}} \frac{\partial^{5} w_{i j}}{\partial \zeta^{4} \partial \tau}+\frac{16 \beta_{i j} g^{*} \bar{L}_{1}^{2}}{15 \kappa_{s}} \frac{\partial^{4} \Psi_{i j}}{\partial \zeta^{3} \partial \tau}+\frac{\beta_{i j} \bar{L}_{2}^{2}}{4 \lambda \kappa_{s}} \frac{\partial^{4} w_{i j}}{\partial \zeta^{4}}-\frac{\beta_{i j} \bar{L}_{2}^{2}}{4 \kappa_{s}} \frac{\partial^{3} \Psi_{i j}}{\partial \zeta^{3}} \\
- & \frac{\beta_{i j} g^{*} \bar{L}_{2}^{2}}{4 \kappa_{s}} \frac{\partial^{4} \Psi_{i j}}{\partial \zeta^{3} \partial \tau}+\frac{\beta_{i j} g^{*} \bar{L}_{2}^{2}}{4 \lambda \kappa_{s}} \frac{\partial^{5} \Psi_{i j}}{\partial \zeta^{4} \partial \tau}-\frac{2 \bar{\Pi}_{0}}{\lambda} \frac{\partial^{2} w_{i j}}{\partial \zeta^{2}}+\frac{1}{\lambda} \frac{\partial^{2} w_{i j}}{\partial \tau^{2}}+\frac{\Gamma_{i j} \bar{\rho} f_{i j}}{\lambda} \frac{\partial^{2} w_{i j}}{\partial \tau^{2}} \\
- & \frac{H_{x}^{* 2}}{\lambda} \frac{\partial^{2} w_{i j}}{\partial \zeta^{2}}+\frac{H_{x}^{*} H_{z}^{*}}{\lambda} \frac{\partial^{2} u_{i j}}{\partial \zeta^{2}}-\Gamma_{i j} \sqrt{\bar{\rho}} f_{i j} \frac{\partial \Psi_{i j}}{\partial \tau}+\frac{\Gamma_{i j} u_{f}^{2} f_{i j}}{\lambda} \frac{\partial^{2} w_{i j}}{\partial \zeta^{2}}+\frac{\partial_{T}^{2} w_{i j}}{\lambda} \frac{\partial^{2}}{\partial \zeta^{2}} \\
& +\frac{\overline{\Delta T} g^{*}}{\lambda} \frac{\partial^{3} w_{i j}}{\partial \zeta^{2} \partial \tau} \frac{\bar{\mu} u_{f}}{\lambda} \frac{\partial^{3} w_{i j}}{\partial \zeta^{3}}-\frac{\sqrt{\bar{\rho} \mu}}{\lambda} \frac{\partial^{2} w_{i j}}{\partial \zeta^{2}}-\left(1-\Gamma_{i j}\right) q_{i 2}^{\prime \text { Pasternak }}-q_{i j}^{\prime}{ }^{v W}=0
\end{aligned}
$$

— for $\delta \Psi_{i j}=0$

$$
\begin{gathered}
-\bar{I}_{i j} \frac{\partial^{2} \Psi_{i j}}{\partial \zeta^{2}}+\bar{I}_{i j} g^{*} \frac{\partial^{3} \Psi_{i j}}{\partial \zeta^{2} \partial \tau}+\beta_{i j} \Psi_{i j}+\frac{\beta_{i j}}{\lambda} \frac{\partial w_{i j}}{\partial \zeta}+\beta_{i j} g^{*} \frac{\partial \Psi_{i j}}{\partial \zeta}+\frac{\beta_{i j} g^{*}}{\lambda} \frac{\partial^{2} w_{i j}}{\partial \zeta \partial \tau}+\frac{\Gamma_{i j} f_{i j} \bar{\rho} u_{f}}{\lambda} \frac{\partial w_{i j}}{\partial \tau} \\
-\bar{h}_{1} \frac{\partial^{2} \Psi_{i j}}{\partial \zeta^{2}}+\frac{\bar{\Pi}_{0}}{\lambda} \frac{\partial w_{i j}}{\partial \zeta}+\bar{I}_{i j} \frac{\partial^{2} \Psi_{i j}}{\partial \tau^{2}}+\Gamma_{i j} f_{i j} \bar{\rho} \frac{\partial^{2} \Psi_{i j}}{\partial \tau^{2}}-\bar{I}_{i j} H_{z}^{* 2} \frac{\partial^{2} \Psi_{i j}}{\partial \zeta^{2}}+\bar{h}_{3} \frac{\partial^{3} w_{i j}}{\partial \zeta^{3}}+\frac{\bar{h}_{4}}{\lambda} \frac{\partial^{3} w_{i j}}{\partial \zeta \partial \tau^{2}} \\
-\frac{2 \beta_{i j} \bar{L}_{o}^{2}}{\kappa_{s}} \frac{\partial^{2} \Psi_{i j}}{\partial \zeta^{2}}-\frac{2 \beta_{i j} g^{*} \bar{L}_{0}^{2}}{\kappa_{s}} \frac{\partial^{3} \Psi_{i j}}{\partial \zeta^{2} \partial \tau}+\frac{2 \bar{I}_{i j} \beta_{i j} \bar{L}_{0}^{2}}{\kappa_{s}} \frac{\partial^{4} \Psi_{i j}}{\partial \zeta^{4}}+\frac{2 \beta_{i j} \bar{I}_{i j} g^{*} \bar{L}_{0}^{2}}{\kappa_{s}} \frac{\partial^{5} \Psi_{i j}}{\partial \zeta^{4} \partial \tau}
\end{gathered}
$$




$$
\begin{aligned}
& +\frac{20 \bar{I}_{i j} \beta_{i j} \bar{L}_{1}^{2}}{25 \kappa_{s}} \frac{\partial^{4} \Psi_{i j}}{\partial \zeta^{4}}+\frac{20 \beta_{i j} \bar{I}_{i j} g^{*} \bar{L}_{1}^{2}}{25 \kappa_{s}} \frac{\partial^{5} \Psi_{i j}}{\partial \zeta^{4} \partial \tau}+\frac{16 \beta_{i j} \bar{L}_{1}^{2}}{25 \kappa_{s} \lambda} \frac{\partial^{3} w_{i j}}{\partial \zeta^{3}}-\frac{32 \beta_{i j}}{25 \kappa_{s}} \frac{\bar{L}_{1}^{2}}{\partial \zeta^{2}} \\
& +\frac{16 \beta_{i j} g^{*} \bar{L}_{1}^{2}}{25 \kappa_{s} \lambda} \frac{\partial^{4} w_{i j}}{\partial \zeta^{3} \partial \tau}-\frac{32 \beta_{i j} g^{*} \bar{L}_{1}^{2}}{25 \kappa_{s}} \frac{\partial^{3} \Psi_{i j}}{\partial \zeta^{2} \partial \tau}+\frac{\beta_{i j} \bar{L}_{2}^{2}}{4 \kappa_{s} \lambda} \frac{\partial^{3} w_{i j}}{\partial \zeta^{3}}-\frac{\beta_{i j} \bar{L}_{2}^{2}}{4 \kappa_{s}} \frac{\partial^{2} \Psi_{i j}}{\partial \zeta^{2}} \\
& +\frac{\beta_{i j} g^{*} \bar{L}_{2}^{2}}{4 \kappa_{s} \lambda} \frac{\partial^{4} w_{i j}}{\partial \zeta^{3} \partial \tau}-\frac{\beta_{i j} g^{*} \bar{L}_{2}^{2}}{4 \kappa_{s}} \frac{\partial^{3} \Psi_{i j}}{\partial \zeta^{2} \partial \tau}=0
\end{aligned}
$$

where $\Gamma_{i j}$ for $j=1$ (inner nanotube) equals 1 and for $j=2$ (outer nanotube) equals 0 . By estimating $l_{0}$ and $l_{1}$, the presented theory converts into the modified couple stress theory.

\section{Numerical method}

DQM is a numerical method like Galerkin's method, Finite Element, Finite Difference method etc. This method is based on the Gaussian integral method and Lagrange polynomial, and some of its important merits are appropriate accuracy and easy access. Partial differential equations will be converted into algebraic equations in this method in two steps. First of all, Chebyshev points distribute grids along the CNTs, then Lagrange polynomial constructs DQ's weighting coefficient matrix (Ghorbanpour Arani et al., 2014). The components of displacement field will be divided into time dependent and time independent functions

$$
u(\zeta, \tau)=u(\zeta) \mathrm{e}^{\mathrm{i} \omega \tau} \quad w(\zeta, \tau)=w(\zeta) \mathrm{e}^{\mathrm{i} \omega \tau} \quad \Psi(\zeta, \tau)=\Psi(\zeta) \mathrm{e}^{\mathrm{i} \omega \tau}
$$

where $\omega=\lambda L \sqrt{\rho_{C N T} / E}$ is the dimensionless natural frequency, $\lambda$ is the natural frequency and $\rho_{C N T}$ is density of the tubes. According to Eqs. (3.1) and (3.2), the equilibrium and boundary equations will change to algebraic equations

$$
\left.\frac{\partial^{n}\left(u_{i j}, w_{i j}, \Psi_{i j}\right)}{\partial \zeta^{n}}\right|_{\zeta=\zeta_{r}}=\sum_{k=1}^{N} C_{r k}^{n}\left\{u_{k}\left(\zeta_{k}\right), w_{k}\left(\zeta_{k}\right), \Psi_{k}\left(\zeta_{k}\right)\right\}
$$

By using Eqs. (3.1), (3.2) and some mathematical manipulations, the partial differential equations turn into state space algebraic equations (Ghorbanpour Arani et al., 2014) which are shown by

$$
\left(\left[\begin{array}{cc}
\mathbf{0} & \mathbf{I} \\
\mathbf{M}_{e}^{-1} \mathbf{K}_{e} & \mathrm{iM}_{e}^{-1} \mathbf{D}_{e}
\end{array}\right]-\omega \mathbf{I}\right)\left\{\begin{array}{l}
d_{d} \\
d^{*}
\end{array}\right\}=\mathbf{0}
$$

where $\mathbf{K}_{e}, \mathbf{D}_{e}, \mathbf{M}_{e}$ and $\mathbf{I}$ are the stiffness, damping, mass and unit matrices, respectively. Subscript $b$ is the element related to the boundary points and subscript $d$ indicates the remaining elements. The imaginary part of the eigenvalue $\operatorname{Im}(\omega) t$ is structural damping and the real part $\operatorname{Re}(\omega)$ represents the natural frequency of the structure.

\section{Discussion}

Some numerical examples and also validation of our results in comparison with other accepted articles are expressed in this Section. Mechanical and geometrical properties of the CNTs are presented in Table 1 (Shen and Zhang, 2011; Zhang and Shen, 2006).

Our purpose is to investigate the natural frequency of the coupled DWCNT. Obviously, it is a nanoscale structure, so small scale theories must be applied in equilibrium equations to obtain appropriate results. 
Table 1. Variety of elasticity and shear modulus of CNTs in different temperatures according to the rolling procedure

\begin{tabular}{|c|c|c|c|c|c|c|}
\hline \multirow{2}{*}{$\begin{array}{c}\text { Rolling } \\
\text { procedure }\end{array}$} & \multirow{2}{*}{$\begin{array}{c}\text { Type of } \\
\mathrm{CNT}\end{array}$} & $\begin{array}{c}\text { Inner } \\
\text { radius } \\
{[\mathrm{nm}]}\end{array}$ & $\begin{array}{c}\text { Thickness } \\
{[\mathrm{nm}]}\end{array}$ & $\begin{array}{c}\text { Tempera- } \\
\text { ture } \\
{[\mathrm{K}]}\end{array}$ & $\begin{array}{c}\text { Young's } \\
\text { modulus } \\
{[\mathrm{TPa}]}\end{array}$ & $\begin{array}{c}\text { Shear } \\
\text { modulus } \\
{[\mathrm{TPa}]}\end{array}$ \\
\hline \hline \multirow{2}{*}{ Zigzag } & \multirow{2}{*}{$(17,0)$} & 0.6654 & \multirow{2}{*}{0.088} & 300 & 3.9 & 1.36 \\
\cline { 5 - 7 } & \multirow{2}{*}{$(21,0)$} & 0.822 & \multirow{2}{*}{0.087} & 500 & 3.89 & 1.36 \\
\cline { 5 - 7 } & & & 500 & 3.81 & 1.37 \\
\hline
\end{tabular}

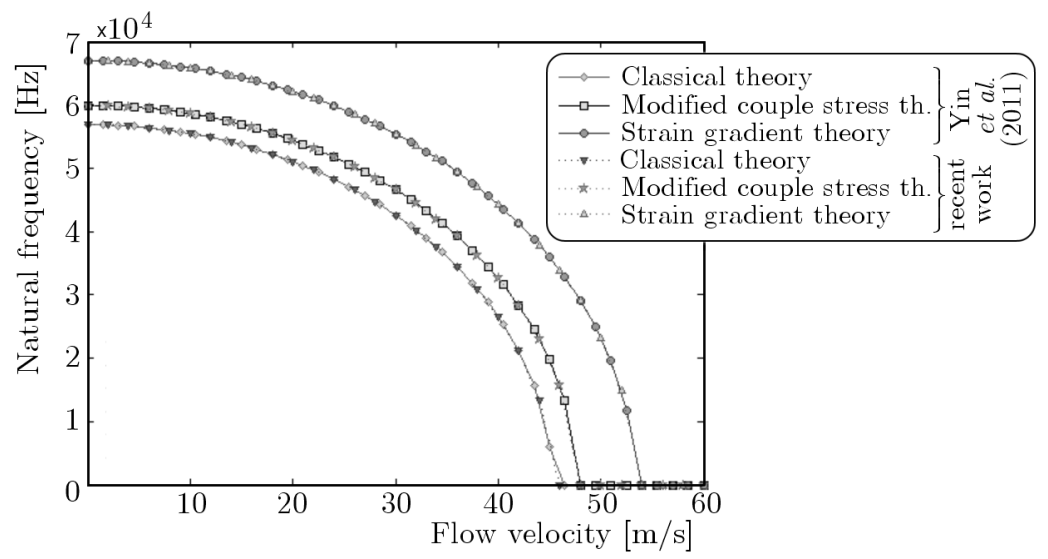

Fig. 2. Comparison of the dimensionless natural frequency with those presented by Yin et al. (2011) for MCST, SGT and classical theories
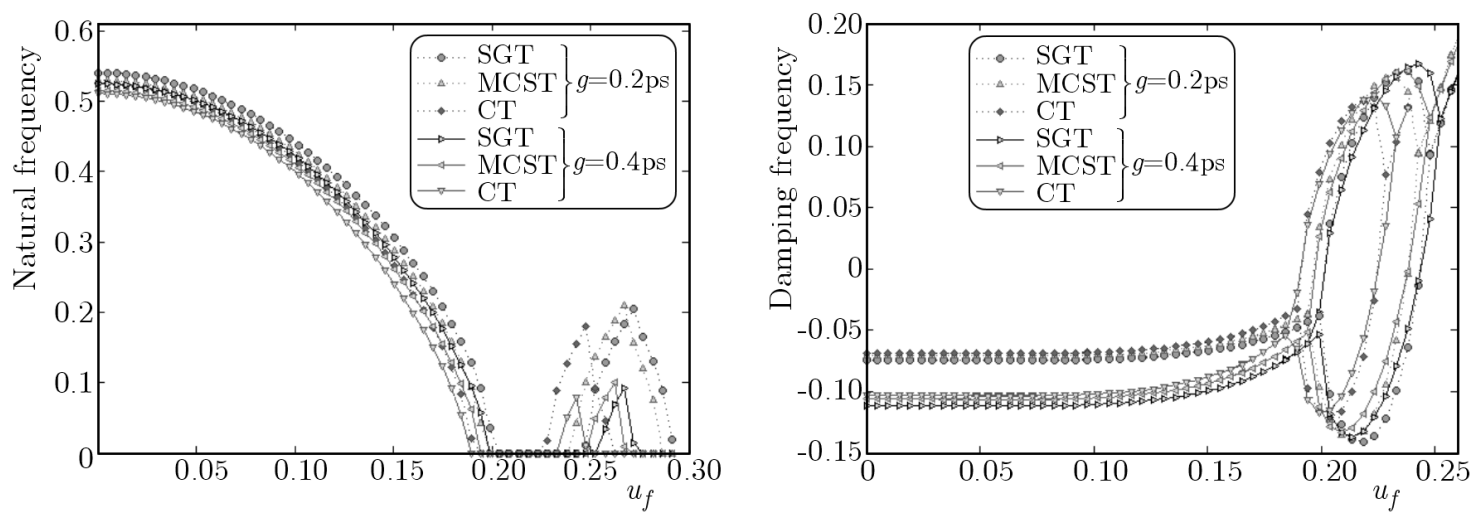

Fig. 3. The effect of structural damping of the CNTs on natural and damping frequencies, $H_{x}=0.1 \mathrm{GA} / \mathrm{m}$ (zigzag inner 17.0 and outer 21.0, clamped-clamped, $L_{0}=L_{1}=L_{2}=0.1 \mathrm{~m}, E_{s} T_{s}=0$, $\left.\tau_{s}=0, H_{z}=0, \Delta T=0\right)$

Figure 2 illustrates the comparison of our results with those by Yin et al. (2011), which is based on modified couple stress theory (MCST), strain gradient theory (SGT) and classical theory $(\mathrm{CT})$, respectively. Figures $3 \mathrm{a}$ and $3 \mathrm{~b}$ present the effects of the Kelvin-Voigt coefficient on the zigzag CNT. Before reaching the critical flow velocity, the natural frequency decreases with an increase in the flow velocity while the damping frequency remains negative and its magnitude decreases. Also the viscoelastic constant increases the magnitude of damping frequency. The effects of magnetic fields on stability of the elastic beam are shown in Figs. 4a and 4b. Figure 4a illustrates that stability of the coupled DWCNT increases as the magnitude of longitudinal magnetic field increases. Figure $4 \mathrm{~b}$ indicates the effect of the transverse magnetic field 
on foundation frequency of the structure. By comparing Fig. 4a with Fig. 4b, we conclude that the longitudinal magnetic field has a more stabilizing effect on the structure than the transverse magnetic field.
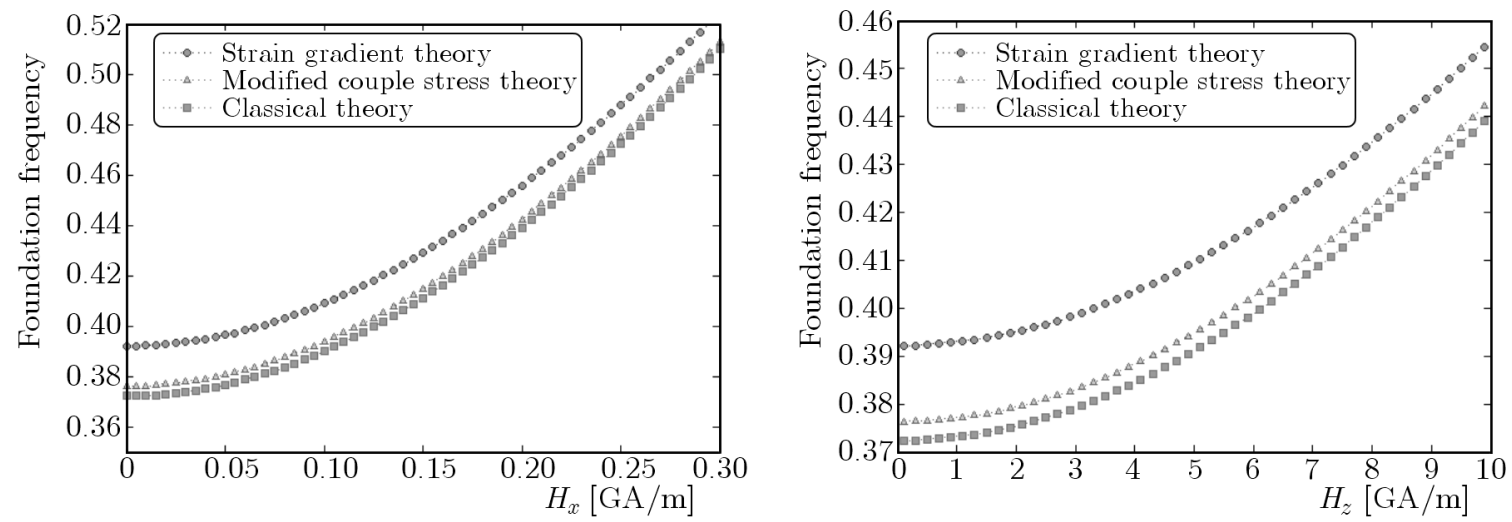

Fig. 4. The influence of 2D-magnetic field on the natural frequency, $\Delta T=200$ and $g^{*}=0$ (zigzag CNT inner 17.0 and outer 21.0, clamped-clamped, $H_{z}=0 \mathrm{GA} / \mathrm{m}, u_{f}=0.01$ )
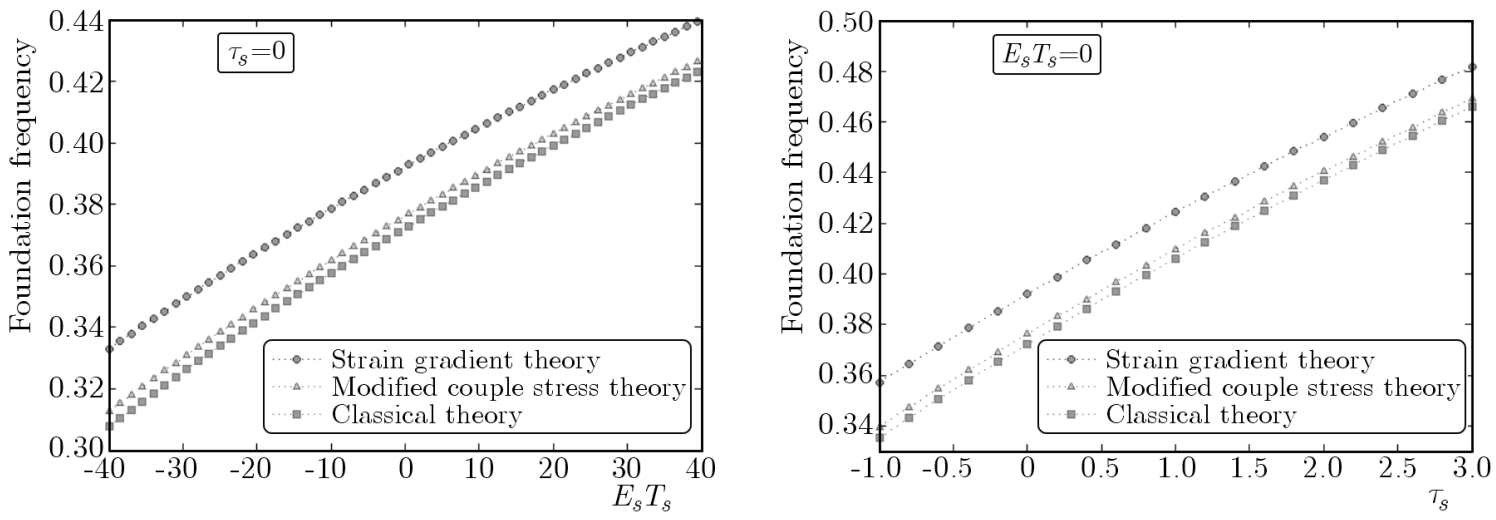

Fig. 5. The effect of surface layer rigidity and layer residual stress constant on the natural frequency $\Delta T=200$ and $g^{*}=0$ (zigzag CNT inner 17.0 and outer 21.0, clamped-clamped, $u_{f}=0.01, H_{x}=0$,

$$
\left.H_{z}=0\right)
$$
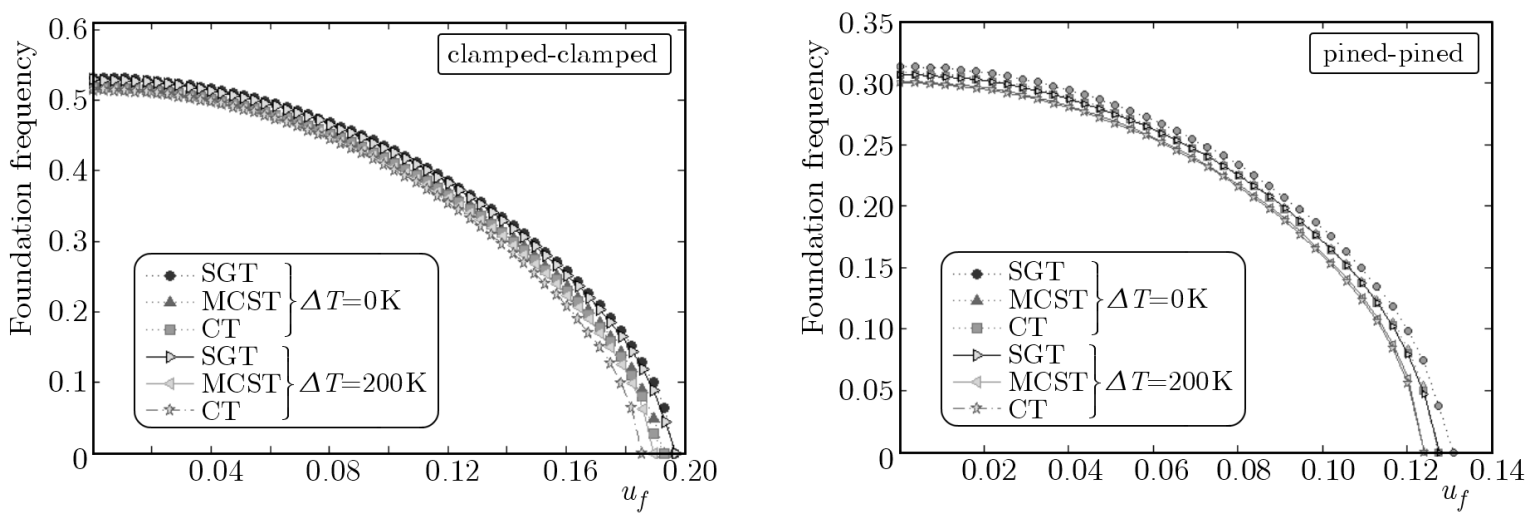

Fig. 6. The effects of different boundary conditions on natural frequency with various temperatures $g^{*}=0\left(\right.$ zigzag inner 17.0 and outer 21.0, $\left.L_{0}=L_{1}=L_{2}=0.1 \mathrm{~m}, E_{s} T_{s}=0, \tau_{s}=0, H_{x}=H_{z}=0\right)$

The surface stresses divide into two parameters, surface layer rigidity $\left(E_{S} T_{S}\right)$ and residual surface tension $\left(\tau^{s}\right)$. The effects of these parameters are shown in Fig. 5a and Fig. 5b, respectively. 
It is obvious that the increasing of the rigidity modulus of the structures reinforces their stability.

Figure 5 demonstrates that the natural frequency of the coupled DWCNT increases with an increase in the surface layer rigidity parameter $\left(E_{S} T_{S}\right)$. It is also shown in Fig. 5 that the increasing of the residual surface tension $\left(\tau^{s}\right)$ increases the natural frequency. Figure 6 illustrates the effects of different boundary conditions and temperatures on the critical flow velocity and natural frequencies. As it can be observed in Fig. 6, strain gradient theory increases the vibration stability region, and the modified couple stress has a more stable region than in the classical theory. It is also shown that the clamped condition is the most stable condition, and the temperature changes have the least effect on the stability of the structure.

\section{Conclusion}

A viscoelastic couple DWCNTs structure based on the TB theory which is affected by the surface stress layer, magnetic field and temperature changes is presented in this paper. In order to obtain an appropriate compliance with the laboratory model, strain gradient and modified couple stress theories are considered in this study. There is a 3\% discrepancy between the stability of CT and other theories. The results reveal that the effect of the viscoelastic coefficient on the vibration response of the system is not negligible. The magnetic field increases the stability region by increasing the rigidity of the system. The effect of the surface stress layer on natural frequency of the structure shows that the surface stress layer is an inherent characteristic for nanostructures. These results are an appropriate guide for designers and engineers to design useful mechanical structures.

\section{Acknowledgement}

The author would like to thank the reviewers for their valuable comments and suggestions to improve the clarity of this study. The authors are grateful to University of Kashan for supporting this work by Grant No. 363443/51. They would also like to thank the Iranian Nanotechnology Development Committee for their financial support.

\section{References}

1. Ansari R., Mohammadi V., Shojaei M. F., Gholami R., Rouhi H., 2014, Nonlinear vibration analysis of Timoshenko nanobeams based on surface stress elasticity theory, European Journal of Mechanics A/Solids, 45, 143-152

2. Ghorbanpour Arani A., Amir S., 2013, Electro-thermal vibration of visco-elastically coupled BNNT systems conveying fl uid embedded on elastic foundation via strain gradient theory, Physica B: Physics of Condensed Matter, 419, 1-6

3. Ghorbanpour Arani A., Amir S., Dashti P., Yousefi M., 2014, Flow-induced vibration of double bonded visco-CNTs under magnetic fields considering surface effect, Computational Material Science, 86, 144-154

4. IıJIma S., 1991, Helical microtubules of graphitic carbon, Nature, 354, 56-58

5. KE L.L., WANG Y.S., 2011, Flow-induced vibration and instability of embedded double-walled carbon nanotubes based on a modified couple stress theory, Physica E: Low-Dimensional Systems and Nanostructures, 43, 1031-1039

6. Khosrozadeh A., Hajabasi M.A., 2012, Free vibration of embedded double-walled carbon nanotubes considering nonlinear interlayer van der Waals forces, Applied Mathematical Modelling, 36, 997-1007

7. KiAni K., 2014, Vibration and instability of a single-walled carbon nanotube in a three-dimensional magnetic field, Journal of Physical and Chemistry of Solids, 75, 15-22 
8. Kong S., Zhou S., Nie Z., Wang K., 2009, Static and dynamic analysis of micro beams based on strain gradient elasticity theory, International Journal of Engineering Science, 47, 487-498

9. Lei X., Natsuki T., Shi J., Ni Q., 2012, Surface effects on the vibrational frequency of double-walled carbon nanotubes using the nonlocal Timoshenko beam model, Composites Part B: Engineering, 43, 64-69

10. Lei Y., Adhikari S., Friswell M. I., 2013, Vibration of nonlocal Kelvin-Voigt viscoelastic damped Timoshenko beams, International Journal of Engineering Science, 66/67, 1-13

11. Lu P., He L.H., LeE H.P., Lu C., 2006, Thin plate theory including surface effects, International Journal of Solids and Structures, 43, 4631-4647

12. Pradhan S.C., Mandal U., 2013, Finite element analysis of CNTs based on nonlocal elasticity and Timoshenko beam theory including thermal effect, Physica E: Low-Dimensional Systems and Nanostructures, 53, 223-232

13. Shen H.S., Zhang C.L., 2011, Nonlocal beam model for nonlinear analysis of carbon nanotubes on elastomeric substrates, Computational Materials Science, 50, 1022-1029

14. Xu M.R., Xu S.P., Guo H.Y., 2010, Determination of natural frequencies of fluid-conveying pipes using homotopy perturbation method, Computers and Mathematics with Applications, 60, $520-527$

15. Yin L., QIAN Q., WAng L., 2011, Strain gradient beam model for dynamics of microscale pipes conveying fluid, Applied Mathematical Modelling, 35, 2864-2873

16. Zhang C.L., Shen H.S., 2006, Temperature-dependent elastic properties of single-walled carbon nanotubes: Prediction from molecular dynamics simulation, Applied Physics Letters, 89, 081904 